\title{
El judo como estrategia pedagógica en el desarrollo de las relaciones sociales de los estudiantes del Liceo Naval
}

\section{The judo as a pedagogical strategy in the development of the social relations of the students of the Liceo Naval}

Lic. Luis Alberto Saltos Aldaz

Unidad Educativa de Fuerzas Armadas Liceo Naval Guayaquil "Cmdt. Rafael Andrade

Lalama”, Ecuador

Lic. Roberto Andrés Rojas Bajaña

Club Deportivo Diana, Ecuador

Evelyn Vianney Saltos Carbo

Universidad de Guayaquil, Ecuador

Autor para correspondencia: saltosalberto@hotmail.com

Fecha de recepción: 23 de julio de 2018 - Fecha de aceptación: 20 septiembre de 2018

Resumen: La idea principal de este proyecto fue mejorar la convivencia escolar a través del Judo. Esta propuesta es una iniciativa presentada por el departamento de Cultura física en coordinación con los departamentos de Inspectoría General y el departamento de Consejería Estudiantil. El plan fue analizar 24 estudiantes entre las edades de 8 a 17 años pertenecientes a diferentes grados y cursos de la Unidad Educativa Unidad Educativa de Fuerzas Armadas Liceo Naval Guayaquil. En este proyecto hemos utilizado estrategias metodológicas de orientación cualitativa: un cuestionario sobre variables sociales e interacciones en el dojo; el diario de clase del profesor y la entrevista a inspectores de curso. Para la realización de esta propuesta se utiliza el paradigma socio crítico enfocado en la "investigación acción" estrategias realizadas que combina la teoría y la praxis y sirve para mejorar el sistema educativo y social. El objetivo de este Proyecto fue Promover la actividad física a través del programa "El judo como estrategia pedagógica" que permitan la solución de conflictos sociales y fortalezcan los procesos de integración y socialización mediante el deporte. La introducción de juegos socializadores de tipo cooperativo en sus distintas variantes y modalidades, fue transversal en todas las partes de la estructura de las sesiones. Los estilos de aprendizaje destacan: resolución de problemas, descubrimiento guiado y mando directo. Finalmente sé logro favorecer la estrategia pedagógica para prevenir conflictos sociales dentro del aula y fuera de esta, promoviendo procesos de integración y socialización a través del deporte de lucha, fundamentada en el ideario original del Fundador del Judo (Jigoro Kano) a la contribución del bienestar social.

Palabras claves: judo; habilidades sociales; estrategias pedagógica e integración

Abstract: The main idea of this project is to improve relationships in the school through Judo. This study is leading the way by different departments such as Physical Culture in coordination with the general inspectors 'office and Students Counseling groups. The study was carried out amoung of 24 students between 8 and 17 ages from different grades and curses at Liceo Naval 
Guayaquil. This investigation was made using methodological strategies of qualitative orientation: a questionnaire about social variables and interactions in the dojo; the field diary of the teacher and the interview. To carry out this proposal, the socio-critical paradigm focused on "action research" is used, strategies that combine theory and praxis and serve to improve the educational and social system. The objective of this Project was to promote physical activity through the program "Judo as a pedagogical strategy" that allows the solution of social conflicts and strengthen the processes of integration and socialization through sport. The introduction of socializing games of cooperative way, using all different variants and modalities, it was transversal in all parts of the structure of the sessions. The learning styles stand out: solving problems, detection guided and direct commanding. Finally it was achieved the pedagogical strategy to prevent social conflicts in and out of the classroom, promoting integration and socialization processes through the sport of combat, based on the original ideology of the Founder of Judo (Jigoro Kano) to the contribution of well-being Social.

Key Words: judo; social competencies; pedagogical strategies; educational facilities; integration

\section{Introducción}

El presente trabajo de investigación es una propuesta del paradigma socio crítico enfocado en la "investigación acción". Aborda el tema de cómo mejorar la convivencia escolar y el progreso del comportamiento, proponiendo estrategias pedagógicas que permitan la solución de conflictos de violencia y agresividad, se hace necesario fortalecer las habilidades sociales de los estudiantes de la Unidad Educativa Unidad Educativa de Fuerzas Armadas Liceo Naval Guayaquil.

La presente investigación, se la realiza a través, de la observación directa y reportes presentados por los departamentos de Inspectoría General (INSGRAL), Departamento de consejería Estudiantil (DECE), y atendiendo las experiencias de los docentes, se ha observado un comportamiento escolar inadecuado, en las diferentes actividades tanto académicas como deportivas.

Por consiguiente, el presente estudio se planteó abordar al judo como estrategia pedagógica que permitan la solución de conflictos sociales y fortalezcan los procesos de integración y socialización mediante el deporte. Cabe destacar que el judo, es encauzado como estrategia sociomotriz para resolver conflictos sociales dentro del aula y fuera de esta, promoviendo procesos de integración y socialización a través del deporte de lucha, basando, del ideario original del fundador del Judo (Jigoro Kano) y su aportación al bienestar social.

Al mismo tiempo, el judo aporta a la comunidad educativa un sistema socioafectiva, que se puede utilizar para insertar calidad de valores sociales en los estudiantes, tratar de mejorar la calidad de vida, de menguar la violencia social, de tener calidad convivencial, mejorar el comportamiento entre pares, y relaciones familiares, orientándose a obtener mejores ciudadanos. Saltos, (2016),"Juegos de lucha e iniciación del Judo en Educación Física" (Trabajo Fin de Master), Universidad Autónoma de Madrid.

Por otra parte, este programa también se enfoca mejorar las habilidades sociales, para Gil Rodríguez, (1995) citado por Ana Betina Lacunza, N. C. (2011), afirma que las "Habilidades sociales influyen en la autoestima, en la adopción de roles, en la 
autorregulación del comportamiento y en el rendimiento académico, entre otros aspectos, tanto en la infancia como en la vida adulta”.

En definitiva, el centro educativo desde su génesis estuvo orientada a apoyar y contribuir a la educación de la niñez y juventud ecuatoriana, basados en los principios de ciencia, honor y disciplina promotores de la cultura de paz, de la interculturalidad, la equidad, la democracia, la ciudadanía, la convivencia social y la integración, basados en el $<<$ Buen Vivir o Sumak Kawsay>>.

El objetivo es, "Promover la actividad física a través del programa el Judo como estrategia pedagógica que permitan la solución de conflictos sociales y fortalezcan los procesos de integración y socialización mediante el deporte"

La presenta investigación se enmarca dentro de la Carta Internacional de la Educación Física y el Deporte de la UNESCO (2015) describen en el art.11. Lo siguiente:

Los programas de deporte en favor del desarrollo y la paz deberían estar encaminados a la erradicación de la pobreza, así como al fortalecimiento de la democracia, los derechos humanos, la seguridad, una cultura de paz y no violencia, el diálogo y la solución de conflictos, la tolerancia y la no discriminación, la inclusión social, la igualdad entre hombres y mujeres, el Estado de derecho, la sostenibilidad, la conciencia ambiental, la salud, la educación y el papel de la sociedad civil.

También, se resalta dentro del artículo 10. De la naturaleza militar de los establecimientos educativos que expresa lo siguiente:

"En el currículo de las unidades educativas militares se considerará una prestación de servicio educativo integral, basado en un alto nivel de desarrollo de valores morales, éticos y cívicos; y un destacado nivel de actividades físicas y deportivas de los estudiantes. Adicionalmente, se considerará el fortalecimiento de la identidad militar de las unidades educativas a fin de contribuir a la formación de los estudiantes en la disciplina, justicia y equidad propias de la vida militar”. Publicado en Orden Ministerial No 158 de 13 agosto 2013".

Así mismo, la Ley del Deporte, Educación Física y Recreación, (2010) en la Décimo Octava disposición general cuyo objetivo además de competir es orientar a “.... generar valores morales, cívicos y sociales y desarrollar fortalezas y habilidades susceptibles de potenciación".

En caso puntual, la propuesta se basa en la disciplina del Judo como una estrategia pedagógica para promover los procesos de integración, socialización, justicia y equidad; siendo su génesis desde las escuelas, colegios y universidades, la cual debe aplicar y fortalecer la coeducación hacia una presencia de relaciones equitativas entre género. De esta manera el proyecto educativo nace de la necesidad de desarrollar habilidades para la vida, por la cual los niños y adolescentes puedan promover formas de convivencias más incluyentes para enfrentar los desafíos que demanda la vida (OPS, 2001). 
En este sentido, existen diversos estudios de casos, como el deporte ha influenciado a mejorar conflictos sociales, promoviendo procesos de integración y socialización en el deporte a través de las artes marciales.

De acuerdo, con lo anteriormente señalado, el Instituto Reação, con el proyecto social de Flavio Canto. Río de Janeiro Brasil (Cantos, 2015) manifiesta que la filosofía del proyecto $<<$ Reação >> se basó en la fortaleza del Judo como una causa fundamental en el desarrollo humano que está enfocado a iniciar el potencial de los niños y adolescentes en las áreas cognitivas, productivas, sociales y personales. El Judo es un deporte olímpico con principios educativos fundamentados en la disciplina, el respeto y la no violencia, constituye una estrategia pedagógica para lograr objetivos establecidos. En este sentido, se considera que la práctica de esta disciplina sirve para que los niños recuperen parte de la autoestima, determinación y confianza en sí mismos.

El Ministerio del Deporte Ecuatoriano y la ONU presentó el programa de Judo "Segura de ti misma" el objetivo es combatir la violencia de género que en los últimos años se ha venido deteriorando por la desacertada actitud machista, en este sentido Andrea Sotomayor, ministra del Deporte indica "no se trata de combatir violencia con violencia, sino es erradicarla con sabiduría y prudencia". Por otro lado el presidente Lenin Moreno (2017) indica que cualquier tipo de "violencia es detestable y en particular cometida hacia la mujer".

Por ello, Weitlauf, Cervone, Smith \& Wright (citados por Tejero, Balsalobre \& Ibáñez 2011), realizaron una investigación con mujeres que practicaban defensa personal quienes acrecentaron su conocimiento y seguridad de las técnicas de defensa personal, también desarrollaron habilidades para enfrentar problemas, con autoeficacia y asertividad.

Cerrando, esta revisión en el aspecto ideológico, el proyecto Judo for Peace (JFP), Judo para la Paz Se basa en los principios filosóficos expuestos en los escritos y conferencias de Jigoro Kano; subraya cuatro elementos claves en el pensamiento de Kano en donde formar judocas-ciudadanos completos va más allá de la actividad física y deportiva:

- Todo individuo debe fortalecer su cuerpo, desarrollar su intelecto y moralidad, y cultivar los aspectos beneficiosos para la sociedad;

- Los ciudadanos deben respetar la sociedad y colaborar en la prosperidad nacional;

- Las personas y las organizaciones deben esforzarse en cooperar para asegurar el mantenimiento de unas relaciones armónicas en la sociedad;

- Respecto a nivel internacional, hay que eliminar todos los prejuicios raciales y promover el avance cultural de modo que las gentes puedan vivir y prosperar mutuamente en comunidad.

\section{Temporalización}

El cronograma del proyecto de judo periodo 2017-2018 del centro educativo, se plantea de la siguiente manera: 


\begin{tabular}{|c|c|c|c|c|c|c|c|c|c|c|c|}
\hline \multicolumn{12}{|c|}{ CRONOGRAMA PROYECTO DE JUDO } \\
\hline \multirow{4}{*}{ ACTMIDADES } & \multicolumn{11}{|c|}{ Periodo Lectivo 2017-2018 } \\
\hline & Jun. & & Jul. & Ago. & Sep. & Oct. & Nov. & Dic. & Ene. & Feb. & \multirow{3}{*}{ Responsables } \\
\hline & Semanas & & Semanas & Semanas & Semanas & Semanas & Semanas & Semanas & Semanas & Semanas & \\
\hline & \begin{tabular}{l|l|l|}
1 & 2 & 3 \\
\end{tabular} & & \begin{tabular}{|l|l|l|}
2 & 3 & 4 \\
\end{tabular} & \begin{tabular}{l|l|l|}
1 & 2 & 3 \\
\end{tabular} & \begin{tabular}{l|l|l|l}
1 & 2 & 3 & 4 \\
\end{tabular} & 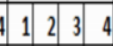 & \begin{tabular}{l|l|l|l|l|}
4 & 1 & 2 & 3 & 4 \\
\end{tabular} & 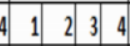 & \begin{tabular}{l|l|l|l|}
1 & 2 & 3 & 4 \\
\end{tabular} & \begin{tabular}{l|l|l|l}
1 & 2 & 3 & 4 \\
\end{tabular} & \\
\hline Ambientación & & & & & & & & & & & Entrenador Judo \\
\hline Pre test (interacción social) & & & & & & & & & & & Entrenador-Psicóloga \\
\hline Visita DECE & & & & & & & & & & & Psicóloga \\
\hline Programación de Judo & & & & & & & & & & & Entrenador Judo \\
\hline Plan de accion: Habilidades Sociales & & & & & & & & & & & Entrenador Judo \\
\hline test. Motores & & & & & & & & & & & Entrenador Judo \\
\hline test. Técnicos & & & & & & & & & & & Entrenador Judo \\
\hline festival de habilidades(Juegos de lucha) & & & & & & & & & & & Entrenador Judo \\
\hline Exhibición & & & & & & & & & & & Entrenador Judo \\
\hline Pos test (interacción social) & & & & & & & & & & & Docente.Psicóloga \\
\hline Ascenso de cinturón blanco-amarillo & & & & & & & & & & & Entrenador Judo \\
\hline
\end{tabular}

Ministerio de Educación

Modificado: Alberto Saltos A.

\section{Material Y Método}

En este programa intervinieron 24 estudiantes de edades comprendidas entre los 8 y 17 años de la Unidad Educativa Liceo Naval Guayaquil. Para cumplir con el compromiso ético, se han usado pseudónimos para proteger las identidades de los sujetos que participaron de este plan, autorización por parte de las autoridades del plantel, consentimiento de los padres de familia para las prácticas de Judo los sábados. INSGRAL-Liceo Naval, (2017).

Tabla 1: participantes

\begin{tabular}{|c|c|c|c|c|}
\hline $\begin{array}{c}\text { Básica Elemental } 6 \text { a } 8 \\
\text { años }\end{array}$ & $\begin{array}{l}\text { Básica Media } 9 \text { a } 11 \\
\text { años }\end{array}$ & $\begin{array}{c}\text { Básica Superior } 12 \text { a } 14 \\
\text { años }\end{array}$ & $\begin{array}{c}\text { Bachillerato } \\
\text { General } \\
\text { Unificado } \\
15 \text { años de edad }\end{array}$ & Total \\
\hline 02 & 04 & 0 & 18 & 24 \\
\hline
\end{tabular}

Las prácticas se llevaron a cabo en una sala de entrenamiento (dojo) con tatami de caucho sintético (1x1m, $50 \mathrm{~mm}$ de espesor), Para ello se ha observado durante ocho meses (un total de 31 clases), programadas los días sábados por la mañana en horarios de $09 \mathrm{H} 00$ a $10 \mathrm{H} 45$ am. Se trabajaron aspectos técnicos de Judo y un plan de acción de habilidades sociales.

\section{Instrumentos para la obtención de información}

En las sesiones de Judo hemos utilizado las siguientes estrategias metodológicas de orientación cualitativa: un cuestionario para la evaluación del estilo de aprendizaje que ha sido adaptado de Baltasar Ramos Gisbert (1999), se eligieron 10 ítems para evaluar las variables sociales e interacciones en el aula de clase; También se utilizó el Diario de clase. (Zabalza, 2004) y las entrevistas para obtener información del proceso. 
Para la realización, de esta propuesta se utiliza el paradigma socio crítico enfocado en la "investigación acción". El término investigación-acción hace referencia a una amplia gama de estrategias realizadas que combina la teoría y la praxis y sirve para mejorar el sistema educativo y social (Torrecilla, 2011).

En el proceso de la propuesta se llevó a cabo en equipo como investigadores externo estuvo a cargo de una profesional en formación de Psicología de la Universidad de Guayaquil. Por otro lado, se coordinó con el departamento de Cultura Física, Inspectoría General y el departamento de Consejería Estudiantil. Además, bajo la dirección técnica de un docente de Judo, que realizaron el proceso reflexivo que vincula la "investigación acción”. Bartolomé, (1986).

\section{Procedimiento}

El sábado 17 de junio del 2017 se inició el proyecto. Al inicio de la programación se realizó un pre-test sobre las interacciones sociales el sábado (24 de junio 2017), luego de esta fecha se aplicó el programa de judo para cinturón blanco amarillo durante el transcurso del proceso. Se realizó una segunda toma de datos pos-test el sábado (18 noviembre 2017) para medir la evolución de la interacción social de los estudiantes.

Tabla 4 Resultados comparativos en porcentaje del Pre y Post Test

\begin{tabular}{cccccc}
\hline Preguntas & \multicolumn{2}{c}{ Pre Test } & \multicolumn{2}{c}{ Post Test } & $\begin{array}{c}\text { \% } \\
\text { SI }\end{array}$ \\
& & NO & SI & NO & Incremento del SI \\
\hline $\mathbf{1}$ & $25,00 \%$ & $75,00 \%$ & $91,67 \%$ & $8,33 \%$ & $\mathbf{6 6 , 6 7 \%}$ \\
$\mathbf{2}$ & $66,67 \%$ & $33,33 \%$ & $16,67 \%$ & $83,33 \%$ & $\mathbf{5 0 , 0 0 \%}$ \\
$\mathbf{3}$ & $70,83 \%$ & $29,17 \%$ & $8,33 \%$ & $91,67 \%$ & $\mathbf{6 2 , 5 0 \%}$ \\
$\mathbf{4}$ & $75,00 \%$ & $25,00 \%$ & $20,83 \%$ & $79,17 \%$ & $\mathbf{5 4 , 1 7 \%}$ \\
$\mathbf{5}$ & $75,00 \%$ & $25,00 \%$ & $12,50 \%$ & $87,50 \%$ & $\mathbf{6 2 , 5 0 \%}$ \\
$\mathbf{6}$ & $29,17 \%$ & $70,83 \%$ & $100,00 \%$ & $0,00 \%$ & $\mathbf{7 0 , 8 3 \%}$ \\
$\mathbf{7}$ & $20,83 \%$ & $79,17 \%$ & $91,67 \%$ & $8,33 \%$ & $\mathbf{7 0 , 8 3 \%}$ \\
$\mathbf{8}$ & $25,00 \%$ & $75,00 \%$ & $91,67 \%$ & $8,33 \%$ & $\mathbf{6 6 , 6 7 \%}$ \\
$\mathbf{9}$ & $37,50 \%$ & $62,50 \%$ & $100,00 \%$ & $0,00 \%$ & $\mathbf{6 2 , 5 0 \%}$ \\
$\mathbf{1 0}$ & $58,33 \%$ & $41,67 \%$ & $100,00 \%$ & $0,00 \%$ & $\mathbf{4 1 , 6 7 \%}$ \\
\hline
\end{tabular}

Elaborado por: Roberto Rojas Bajaña

Revisado por: Autores 


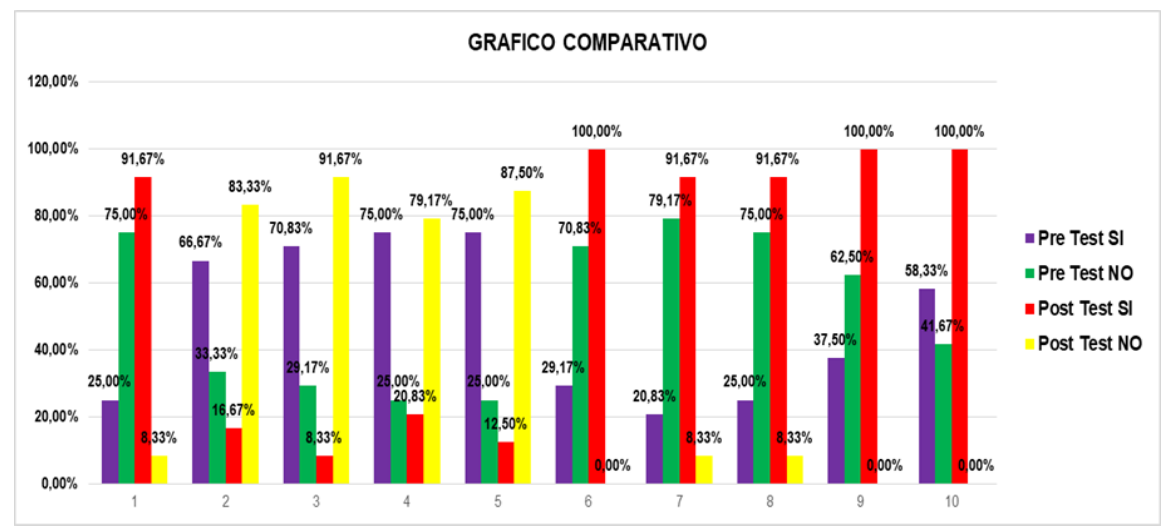

Elaborado por: Roberto Rojas Bajaña- Revisado por: Autores

Resultados Y Discusión

\section{Análisis de los datos}

La obtención de la información del proyecto se realizó con el objetivo de conocer la interacción social en la sala de judo.

Se realizó un pre test en la fase inicial (cuarta sesión), Los datos de la tabla 4 e ítems 1 muestran los valores obtenidos de forma negativa el 75\% los estudiantes no saben "fomentar la convivencia y cooperación de la mejora en relaciones afectivas del grupo". Por otro lado, en la ítems 8 se demuestra con un porcentaje del 75\% los estudiantes no "colaboran con las actividades grupales y respetan las aportaciones de los demás y las normas establecidas". Asimismo, en la pregunta 9 se verifico con el 62,50\% los estudiante no "trabajan en grupo no es respetado y ayudado por los compañeros".

Del mismo modo se aplicó el pos test en la fase final, a través del cuestionario de interacciones sociales. Los resultados obtenidos después de la aplicación del programa de judo indican lo siguiente datos.

Los datos de la tabla 4 y pregunta 1 muestran los valores obtenidos de forma positiva el $91,64 \%$ los estudiantes sí "fomentan la convivencia, la cooperación y la mejora de las relaciones afectivas en el grupo". Por otro lado, en la pregunta 8 se demuestra con un porcentaje del $91,67 \%$ estudiantes si "colaboran con las actividades grupales y respetan las aportaciones de los demás y las normas establecidas". Asimismo, en la pregunta 9 se verifico con el 100\% que los estudiante cuando "trabaja en grupo es respetado y ayudado por los compañeros". Tomando como referencia estos resultados, se demuestra que durante el proceso, los cambios fueron muy significativos y que las actividades físicas en sí, permitieron un cambio sustancial del comportamiento social, (la estrategia pedagógica y el plan de acción si incidió significativamente).

Con esto, podemos afirmar que el judo es una estrategia pedagógica que permite una adaptación en el ámbito educativo y formativo, además los estudiantes demostraron autoeficacia en el comportamiento. La autoeficacia para el psicólogo Albert Bandura (2017), es la creencia que tiene el ser humano de ser capaz de realizar con éxito alguna actividad.

Cabe destacar, estudios realizados por Navarro, Montesino, Celis, Jaimes y Palomino (2014) manifiestan que "el Judo es una actividad física que permite a los sujetos re direccionar 
sus comportamientos sociales e incrementar una socialización con sus compañeros así como sus semejantes".

Asimismo, en el proyecto Tatami 33. De Montevideo Uruguay se promovieron estrategias que reducen la situación de exclusión y marginación social de niños y niña entre 8 y 12 años. Los niños participantes han avanzado en su rendimiento escolar significativamente y mejorado considerablemente en su conducta y en su integración social. LAGUARDA, (2007).

En suma se comprobó que los aspectos teóricos de los artículos analizados y puestos en práctica sobre el Judo cumplen una considerable función en la afectividad; limita los conflictos sociales, refuerza la integración y socialización, además brindaron de base científica al presente estudio y se elaboraron contenidos del Judo sobre las estrategias pedagógicas adecuadas para los estudiantes.

\section{La observación en clase}

Se utilizó el diario de clase; de manera que se plasmó las vivencias y práctica de los estudiantes los días sábados, en síntesis, lo que se extrae de las observaciones es lo siguiente:

En las primeras sesiones (1-2) asistieron los padres de familia especialmente de los niños del Básico elemental y muy poco los del Bachillerato General Unificado, se realizó un diagnóstico en las sesiones, los sujetos analizados demostraron pocos hábitos de atención al docente, no pedían permiso para salir del tatami se entretenían con la mirada de los padres presentes, otros solo querían correr y no prestaban atención a las indicaciones del docente, luego en la parte principal de clase se realizó una explicación sobre el judo, su filosofía, ayuda mutua y prosperidad mutua, el reglamento y los valores que aporta el respeto, la solidaridad, la honestidad; como seguimiento de esta actividad, los estudiantes les llamo mucho la atención de practicar los juegos de lucha, en la parte final se saludó para despedir la clase, sin embargo, algunos estudiantes interrumpían y no seguían las indicaciones del profesor.

En la tercera sesión se realizaron las prácticas dialogando sobre el respeto al profesor, al compañero, mediante acciones como el saludo o equivalentes, algunos estudiantes especialmente los niños se desconcentraban fácilmente de las actividades propuestas (juegos de lucha), se practicó el juego para observar el comportamiento de cada estudiante, se observó que incumplían las normas en algunos casos excluían a sus compañeros del juego.

Según el cronograma planteado se realizó en la cuarta sesión, el análisis de los estudiantes a través del cuestionario de interacciones sociales (pre test) con el fin de valor el comportamiento.

Volviendo a las prácticas de la quinta sesión se observa que la mayoría de los estudiantes alteran la convivencia; presentan una carencia de habilidades sociales. Continuando, con la clase se realizaron ejercicios de caídas en el colchón quitamiedos, algunos estudiantes no respetan la formación y siempre querían estar primeros para efectuar los ejercicios, ocasionando el descontrol en la clase se terminó la clase dialogando y reflexionando sobre el comportamiento de los estudiantes, luego siguiendo el plan de acción se escogió a un 
estudiante para cumplir con la lectura sobre el respeto y se pidió la opinión de los participantes, las ideas expuestas, fueron muy significativas. Se formó y se efectuó el saludo de judo.

Durante las siguientes sesiones: con los resultados obtenidos en el pre test se fortaleció el plan de acción de valores para aplicarlo en todas las prácticas durante diez minutos clase, efectuando conversatorios con los estudiantes para promover actitudes de compañerismo y colaboración entre comunidad educativa: desarrollando valores como la ayuda mutua, la entrega, la tolerancia, la cooperación, la paz, la responsabilidad, amistad etc. (se realizó la retroacción al finalizar la clase)

A lo largo, de las demás sesiones se realizó el trabajo específico de técnicas de judo se aplicaron juegos direccionados a la lucha, "Jugado sin hacer daño", inculcando el respeto de las reglas y los principios del juego limpio, en la parte principal de la clase se realizaron caídas y técnicas de proyección desde la posición de rodillas, los estudiantes comprenden que aparte de responsabilizarse de la integridad física, de sus compañeros, son responsables de su aprendizaje, por último, se terminó realizando un juego cooperativo se evidencio el valor de la solidaridad al trabajar en equipo, ya los estudiantes colaboran entre sí. (Se habló sobre responsabilidad)

Asimismo, en el proceso técnico de judo se participó en una exhibición de Judo en el mes octubre donde se observó que el $80 \%$ de los logros alcanzados en el orden técnico, físico y educativo fueron positivos, para tal efecto, han avanzado significativamente en el comportamiento. De igual manera, expresan respeto en el triunfo y la derrota, también se observó el espíritu de lucha demostrando honestidad y buena convivencia.

En el mes de noviembre se realizó la toma del pos test, donde ya se evidencio el progreso en las relaciones sociales de los estudiantes observando actitudes de compañerismo y colaboración entre los alumnos y valores como la ayuda, la entrega, el auto superación, y la autoeficacia. En las diferentes prácticas del judo se destacan un gran fomento de las habilidades sociales entre los estudiantes, los randoris (competencias), existe un ambiente favorable aceptan sus errores cuando comenten alguna infracción, se llega al dialogo para llegar algún acuerdo.

Como se ha mostrado, el diario de clase del profesor; ha sido un escrito personal de suma importancia para el docente al realizar interpretaciones, reflexiones y explicaciones e información significativa, durante las sesiones planificadas ya que, según Zabalza, (2004) es un instrumento de registro del proceso de aprendizaje, que aportan información de lo que sucede en nuestras clases y cómo ha evolucionado, es una "visión de primera mano" En definitiva, se puede indicar que los deportes, la actividad física y en concreto el judo, es una herramienta pedagógica que ayuda a mejorar el comportamiento de los infanto-adolecentes, a desarrollar habilidades sociales, y a mejorar la calidad de vida.

\section{Análisis del diario}

En las primeras sesiones el comportamiento de los alumnos fue desatento ya que mostraban pocos hábitos de atención al docente y de respeto al área de entrenamiento, sin embargo se mostraban interesados cuando se les explicaba las actividades, al momento de 
ejecutarlas algunos excluían a sus compañeros o irrumpían las normas propuestas, se mostraban distraídos y carentes de habilidades sociales lo que fue mejorando al avanzar las sesiones sobre todo cuando se implementó el plan de acción de valores en donde se tomaron 10 minutos de conversación en cada sesión.

Al impartir los trabajos específicos técnicas de Judo y juegos de oposición aportó, a fomentar el respeto a las reglas y al juego limpio, además de hacerse responsable de la integridad física de sus compañeros. Se fomentó el juego cooperativo con el fin de reforzar la solidaridad y el trabajo en equipo de los alumnos teniendo resultados bastante positivos, incluso el conjunto de todo este accionar permitió que el alumno aceptara el triunfo y la derrota, además de ser autoeficases. Lo observado en la programación demuestra que los parámetros fueron captados e interiorizados de modo constructivista por los estudiantes, practicando técnicas de Judo y participando en juegos de habilidades (combates) los estudiantes aplicaron en las sesiones el "Jugar sin hacer daño", respetaron las reglas y los principios del juego limpio. Del mismo modo se logró observar actitudes de compañerismo y colaboración entre sus pares empleando valores como la ayuda mutua, auto superación y la entrega.

Como recomendaciones se sugiere realizar este tipo de vivencias a través de estrategias pedagógicos a través actividades académicas-deportivas, ya que permite mejorar el clima motivacional de los alumnos e incluida la comunidad educativa. Por otra parte, Zabalza (2004), en relación, con el uso del diario del profesor recoge información significativa del proceso enseñanza aprendizaje; destaca describir momentos; identificar problemas que se hallan ido presentando, se profundiza en la comprensión del significado y posibilita la toma de decisiones. Otra forma de contribuir es la propuesta por Ospina, D. (2017), quien manifiesta, que el uso del diario de clase, es una estrategia didáctica de gran importancia atendiendo estas consideraciones. Siguiendo esta línea del diario como un instrumento personal, Zabalza (2004), manifiesta, reiteradamente; los docentes que han trabajado con diarios son muy útiles para provocar la reflexión y el mejor conocimiento de uno mismo y de sus acciones.

Por otra parte, una de las limitaciones de la investigación fue la poca asistencia a las prácticas de judo los días sábados por parte de los miembros del DECE, en su mayoría no podía asistir por diferentes actividades, sin embargo en la jornada académica ordinaria si realizaban el seguimiento y control a los alumnos, cabe destacar que la colaboración de los inspectores (INSGRAL) fue de gran importancia ya que nos permitía saber la evolución de los alumnos dentro de la jornada académica.

\section{Análisis de las entrevistas}

Con la finalidad, de conocer percepciones y apreciaciones sobre la investigación relacionada con el comportamiento. Realizaremos esta entrevista semi estructurada de final abierto, que es parte necesaria para este estudio, el lugar donde se han realizado las entrevistas Liceo Naval Guayaquil con el aval de los encuestados a través de una carta de consentimiento.

Un Inspector de curso, considera que él cambio fue sustancial en el comportamiento y de gran valor, el proyecto de Judo considera que es una estrategia pedagógica, apoyo a la convivencia escolar porque limita los conflictos sociales, reforzando la integración y socialización. 
Además, manifiesta el entrevistado, que la adolescencia es la mejor época de la vida es la etapa en donde se fortalece la inteligencia se desarrolla nuevos aprendizajes. Por otra parte, han cambiado su forma de comportarse por la globalización son más liberales, más irresponsables, tienen otra forma muy diferente de ver la vida. Finalmente, indica que el Judo es un deporte que fomenta desde que se empieza a practicar el respeto por los demás, el juego limpio, la honestidad y la humildad.

A este respecto, hay muy buenos resultados con la participación del programa, se evidencia mejoría, en la convivencia, hay una mayor integración socialización y respeto de sus pares y en la comunidad educativa, esto es un proceso que se debe continuar con el apoyo de los padres, el centro educativo y el medio donde viven. En conclusión sugiere que los educadores de otras áreas deben proponer estos proyectos que ayuden a mejorar la conducta.

Por otro lado el DECE a través, una Psicóloga educativa cree que ha cambiado las actitudes y las formas de vida de los adolescentes en los últimos años de vida; manifiesta que la causa es el mayor acceso a la tecnología sin un control y uso adecuado. De ahí que, se considera que las redes sociales han contribuido para ser un "arma de distracción masiva".

En referencia a los deportes, para el desarrollo de las habilidades sociales, indica que sí, hay una interacción directa. Por otra parte, se le pregunta: ¿Usted considera que los deportes de combate son violentos? Expresa: no todos los deportes son violentos; en este caso el judo utiliza estrategias no agresivas, siendo más bien defensivas, por consiguiente a través de esta propuesta permitió fortalecer las habilidades sociales, y el manejo de las emociones de los estudiantes participantes de este proyecto.

Para concluir, se le pregunto: ¿Cómo se manejó las habilidades sociales en sus estudiantes que participaron en el proyecto de judo? Seguidamente expresa: que hubo resultados positivos, sin embargo en los niños, hay aspectos que por su propia edad son variables

\section{Conclusión}

El programa de intervención que se aplicó en la Unidad Educativa Liceo Naval de Guayaquil "Cmdt. Rafael Andrade Lalama". Logró mejorar el comportamiento escolar de los estudiantes mediante la aplicación del programa "El judo como estrategia pedagógica en el desarrollo de las habilidades sociales".

Este programa fue motivante porque en la evaluación final de ascensos obtuvieron el cinturón amarillo por sus méritos y conocimientos adquiridos como: modales, normas de higiene y seguridad, dominando globalmente los fundamentos básicos del <<Judo suelo y Judo pie >.

Finalmente el proyecto cumplió el objetivo de: promover la práctica de Judo también como una estrategia afectiva porque, se logró mejorar la calidad de vida, menguar la violencia social, de tener calidad convivencial, y optimizar el comportamiento, otra tarea prioritaria, fue de obtener mejores ciudadanos. 


\section{Bibliografía}

Ana Betina Lacunza, n. C. (2011). Las habilidades sociales en niños. Obtenido de http://www.redalyc.org/pdf/184/18424417009.pdf

Baltasar, g. (1999). Cuestionario para la evaluación del estilo de aprendizaje para alumnos de e. Primaria. Obtenido de http://paidos.rediris.es/needirectorio/tema3.htm

Bandura, a. (2017). Que es la autoeficacia-Albert Bandura. Obtenido de http://www.laautoestima.com/que-es-el-poder-de-la-autoeficacia.html

Bartolomé. (1986). La investigación cooperativa. Obtenido de http://educar.uab.cat/article/viewfile/462/443

Bernardino Javier Sánchez-Alcaraz Martínez 1, b. M.-m. (2018). Observación de las conductas que alteran la convivencia en las clases de educación física y relación con la violencia escolar cotidiana. Obtenido de

http://revistadeeducacionfisica.com/articulo/observacion-de-lasconductas-que-alteran-laconvivencia-en-las-clases-de-educacion-fisicay-relacion-con-la-violencia-escolarcotidiana-2371-sa-h5a60abf281f2a

Cantos., f. (2015). Proyecto social judo. Obtenido de ightland.vice.com/.../elinstituto-reacao-elproyecto-social-de-flavio-canto-en-ro-de-j...

Carriedo, a. (28 de noviembre de 2012). Fectos de distintos tipos de entrenamientos por contraste sobre la capacidad $d$. Obtenido de https://www.researchgate.net/profile/alejandro_carriedo/publication/2597 _benefits_of_physical_education_in_children_diagnosed_with_attention_deficit_hyperac tivity_disorder_adhd/

Comisión ecuménica de derechos humanos (cedhu). (01 de enero de 2018). 151 femicidios en ecuador durante el 2017, registró cedhu. Obtenido de https://www.eluniverso.com/noticias/2018/01/01/nota/6546565/151femicidios-ecuadordurante-2017-registra-cedhu

Ecuador, c. R. (2008). Cultura física y tiempo libre. Obtenido de http://www.oas.org/juridico/pdfs/mesicic4_ecu

Edmodo. (2017). Obtenido de https://www.edmodo.com/home

Español, e. E. (2016). Cinturón negro de judo en ciudad de dios. Obtenido de http://www.elespanol.com/deportes/juegosolimpicos/20160507/122987849_0.html

Estilos de aprendizajes. (26 de marzo de 2018). Obtenido de https://www.ecured.cu/estilos_de_aprendizaje

Fundar. (2009). Proyecto de integración socioeducativa a través del judo. Obtenido del comité de Meurthe-et-Moselle también puso en marcha recientemente la operación 
Gil rodríguez, f. L. (1995). Habilidades sociales y salud. Madrid: pirámide

Gisbert, b. R. (1999). Cuestionario para la evaluación del estilo de aprendizaje para alumnos de e. Primaria. Obtenido de http://paidos.rediris.es/needirectorio/tema3.htm

Insgral-liceo naval. (19 de mayo de 2017). Circular no licgua-insgral2017-019-0 Guayaquil...liceo naval. Obtenido de http://www.liceonaval.mil.ec/archivos/ensayosyudo17.pdf

Judo, f. M. (2010). Judo una visión diversa. Visión libros. Obtenido de /books.google.com.ec/books?id=ar79z_tmmqic\&pg=pa302\&lpg

La ley del deporte, e. F. (2010). Obtenido de http://www.deporte.gob.ec/wpcontent/uploads/downloads/2015/03/ley-del-deporte.pdf

Laguarda, g. (2007). Tatami: un proyecto social con mucho corazón y escaso apoyo empresarial. Obtenido de http://www.lr21.com.uy/comunidad/284176-tatami-un-proyecto social conmucho corazón-y-escaso-apoyo-empresarial

Mendo, m. A. (octubre de 2013). La metodología observacional en el ámbito del deporte. Obtenido de http://www.redalyc.org/html/865/86528863001/

Moreno, 1. (24 de noviembre de 2017). Lenin moreno: no podemos permitir que en ecuador muera una mujer cada 4 días. Obtenido de https://www.eluniverso.com/noticias/2017/11/24/nota/6495865/leninmoreno-nopodemos-permitir-que-ecuador-mujer-muera-cada-

Nacional, b. V. (2017). Objetivo 3. Obtenido de http://www.buenvivir.gob.ec/objetivo-3.-mejorarla-calidad-de-vida-de-lapoblacion

Navarro, m. 1. (2014). Obtenido de www.academia.edu/.../eljudo como estrategia terapéutica en el desarrollo de navarro, m. M., \& navarro, montesinos, Celis, Jaime, palo. (21 de septiembre de 2015). El judo como estrategia terapéutica en el desarrollo de las relaciones sociales de los niños y adolescentes. Obtenido de https://judocamv.wordpress.com/2015/09/21/el-judo como-estrategiaterapeutica-en-eldesarrollo-de-las-relaciones-sociales-de-los-ninos-yadolescentes/comment-page-1/

OMS. (2017). La actividad física en los jóvenes. Obtenido de www.who.int/dietphysicalactivity/factsheet_young_people/es/

OPS. (2001). Enfoque de habilidades para la vida para un desarrollo saludable de niños y adolescentes. Obtenido de convivencia.files.wordpress.com/2008/11/habilidades2001oms65p.pdf

Oquendo, j. (2014). "el judo como mediador del clima escolar deteriorado". Obtenido de http://repositorio.pedagogica.edu.co 
Ospina, d. (2017). El diario como estrategia didáctica. Obtenido de http://aprendeenlinea.udea.edu.co/boa/contenidos.php/8ffccad7bc2328 a00d9344288580dd7/128/1/contenido/

P., d. P. (2016). El diario como estrategia didáctica. Obtenido de http://aprendeenlinea.udea.edu.co/boa/contenidos.php/8ffccad7bc2328a a00d9344288580dd7/128/1/contenido/

Ramos, b. (1999). Recursos educativos. Obtenido de cuestionario para la evaluación del estilo de aprendizaje : http://paidos.rediris.es/needirectorio/tema3.htm

Saltos, a. (2016). Juegos de lucha e iniciación al judo en educación física. Guayaquil, guayas, Ecuador.

Saltos, a. (2016). Tfm. Juegos de lucha e iniciación al judo en educación física. Guayaquil, Ecuador

Saltos, a. (2017). Educación física. Obtenido de http://saltosluisalberto.blogspot.com/

Tejero-González, c., \& balsalobre-fernández, c. I.-c. (Septiembre de 2011). La defensa personal como intervención educativa en la modificación de actitudes violentas. Obtenido de http://www.redalyc.org/html/542/54222177005/

Torrecilla, f. J. (2011). Investigación acción-universidad autónoma de Madrid. Obtenido de

https://www.uam.es/personal_pdi/stmaria/jmurillo/investigacionee/presentaciones/curso_ 10/inv_accion_trabajo.pdf

Unesco. (2015). Carta internacional revisada de la educación física, la actividad física y el deporte. Obtenido de http://portal.unesco.org/es/ev.phpurl_id=13150\&url

Universidad pedagógica de Durango. (2007).indicadores párala elaboración y evaluación de proyectos. Obtenido de Http://www.upd.edu.mx/pdf/varios/indicadoreselaboracionevaluacion.ppf upj. (9 de nov de 2017). "segura de ti misma" proyecto del judo ecuatoriano. Obtenido de http://panamericanjudo.com/site/admin/segura-de-ti-mismaproyecto-del-judo-

ecuatoriano-que-busca-empoderar-a-la-mujer/villamón, m. (2005). Introducción al judo. En m. Villamón, introducción al judo. Hispano europea.

Vivir, b. (2017). Mejorar la calidad de vida de la población. Obtenido de http://www.buenvivir.gob.ec/objetivo-3.-mejorar-la-calidad-de-vida-de-lapoblacion

Vivir, p. N. (2017). Obtenido de www.buenvivir.gob.ec/objetivo-3.-mejorar-lacalidad-de-vida-dela-poblacion

Zabala, m. A. (2004). Diario de clases un instrumento de investigación y desarrollo profesional. Madrid: Narcea s.a. De ediciones. 